\title{
Printable Organic Electronic Materials for Precisely Positioned Cell
}

\author{
Attachment \\ Jeffrey A. Horowitz, Xiaoyang Zhong, Samuel J. DePalma, Maria R. Ward Rashidi, Brendon M. \\ Baker, Joerg Lahann, Stephen R. Forrest* \\ Jeffrey A. Horowitz, Prof. Stephen R. Forrest* \\ Department of Electrical and Computer Engineering, University of Michigan \\ 1301 Beal Ave, Ann Arbor, MI 48109, United States \\ E-mail: stevefor@umich.edu
}

Xiaoyang Zhong, Maria R. Ward Rashidi

Department of Materials Science and Engineering, University of Michigan

2800 Plymouth Rd, Ann Arbor, MI 48109

Samuel J. DePalma, Prof. Brendon M. Baker

Department of Biomedical Engineering, University of Michigan

2200 Bonisteel Blvd, Ann Arbor, MI 48109

Xiaoyang Zhong, Maria R. Ward Rashidi, Prof. Joerg Lahann

Biointerfaces Institute, University of Michigan

2800 Plymouth Rd, Ann Arbor, MI 48109 


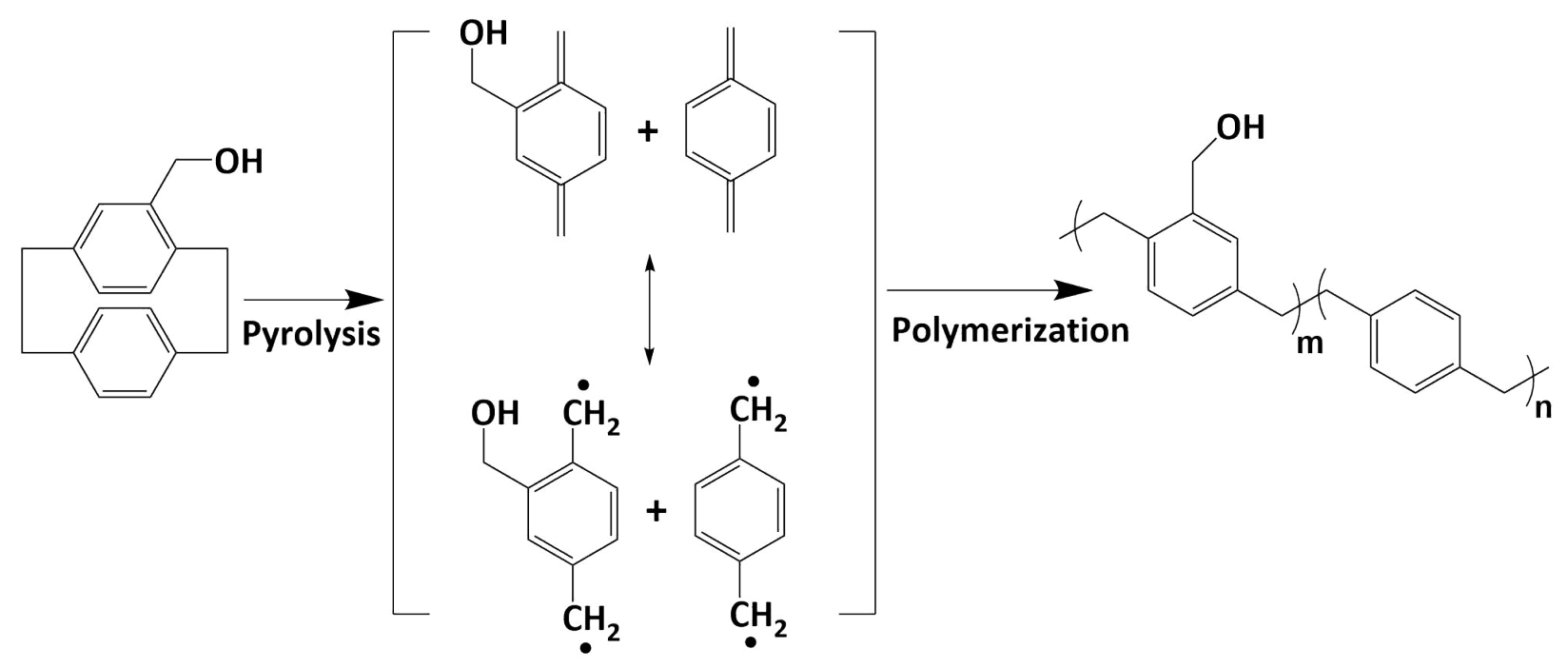

Supplementary Figure 1. Schematic of the polymerization of 2hydroxymethyl[2.2]paracyclophane. 

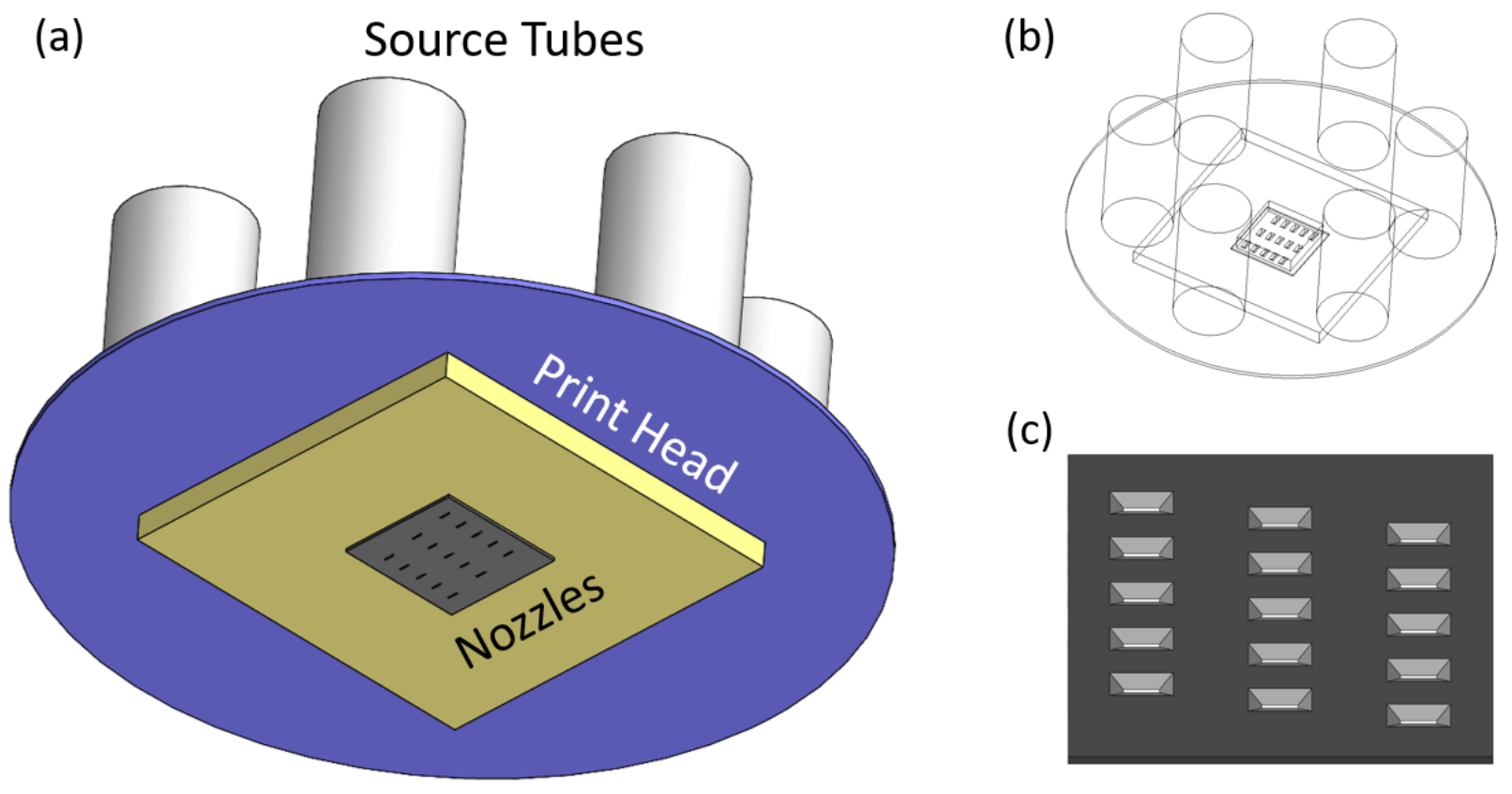

(c)

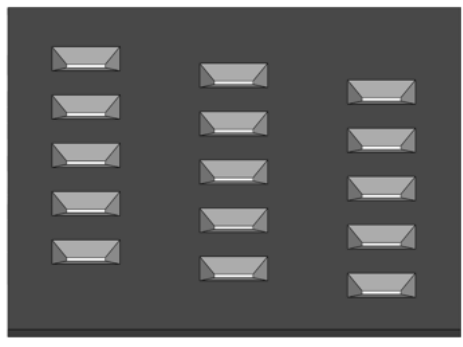

Supplementary Figure 2. Diagrams of the main aspects of the organic vapor jet printing (OVJP) setup. (a) 3D rendering of the OVJP print head. Organic material is picked-up and carried by $\mathrm{N}_{2}$ gas through source tubes and a microfluidic manifold (blue circle) to a Kovar ${ }^{\circledR}$ print head (gold rectangle) which channels the gas mixture to a silicon micronozzle array. (b) A wireframe drawing of the OVJP apparatus. (c) A drawing of a silicon micronozzle array. Nozzles are formed by $\mathrm{KOH}$ etching such that they are tapered at an angle of $54.7^{\circ}$. 


\section{Spectroscopic analysis}

The PEGMA polymer brush growth process is analyzed using XPS and FTIR. The XPS results in Figure S2 compare the presence of $\mathrm{Br}$ for the different reaction steps. After the initial deposition of DBP, CBP, or PPX-HM, there is no $\mathrm{Br}$ on the samples (Figure S2a). Following the attachment of the acetyl bromide in the ATRP initiator reaction, two characteristic $\mathrm{Br}$ signals are visible on samples coated with PPX-HM, but not on the samples coated with DBP or CBP (Figure S2b). This indicates successful covalent immobilization, rather than non-specific adsorption. Following the ATRP reaction, $\mathrm{Br}$ is ultimately consumed during extended chain propagation, and the two Br signals are no longer visible in the XPS spectra (Figure S2c). These results demonstrate that acetyl bromide groups are selectively formed on PPX-HM surfaces. Because PEGMA polymer brushes require initiation from these groups, the polymer brushes can be patterned selectively onto PPX-HM patches, and cannot form on surfaces covered by the small molecule compounds. The corresponding FTIR spectra confirm the chemical reaction scheme used to modify the PPX-HM films. The characteristic bands of the PPX-HM film (Figure S2d) include $\mathrm{OH}\left(3360 \mathrm{~cm}^{-1}\right)$ and $\mathrm{C}-\mathrm{H}\left(2846,2915,2962 \mathrm{~cm}^{-1}\right)$ stretching vibrations. After the ATRP initiator immobilization, the $\mathrm{C}=\mathrm{O}\left(1731 \mathrm{~cm}^{-1}\right)$ and $\mathrm{C}-\mathrm{O}-\mathrm{C}\left(1159 \mathrm{~cm}^{-1}\right)$ stretching vibrations are observed (Figure S2e), indicating successful reaction of the hydroxyl groups of the PPX-HM films with the acetyl bromide groups of the ATRP initiator. After the ATRP initiator reaction, bands indicative of the C-O-C stretching vibrations are broadened and a slight shift to higher wavenumbers is observed (Figure S2f). ${ }^{1}$ 
(a)

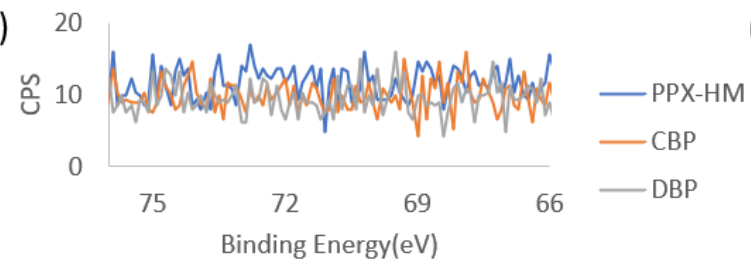

(b)

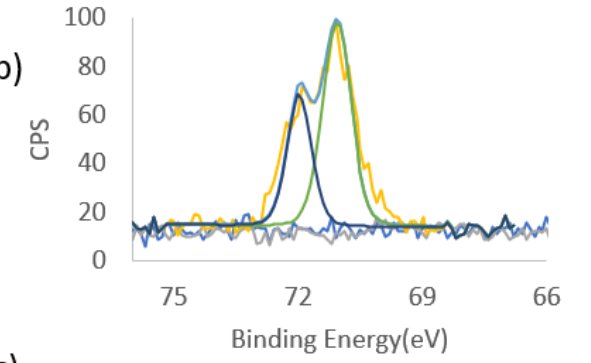

(c)

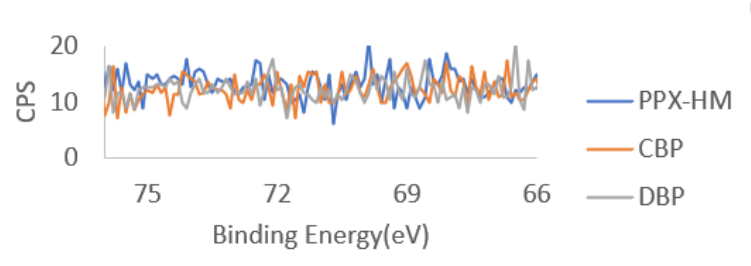

(d)

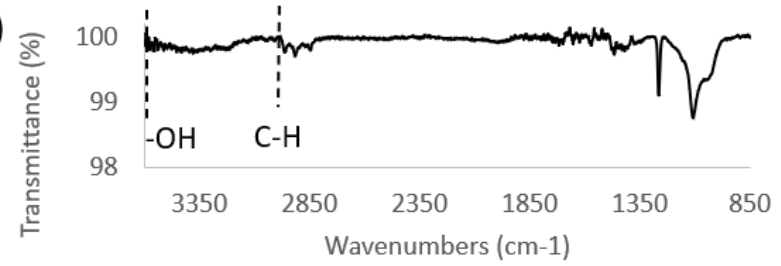

(e)

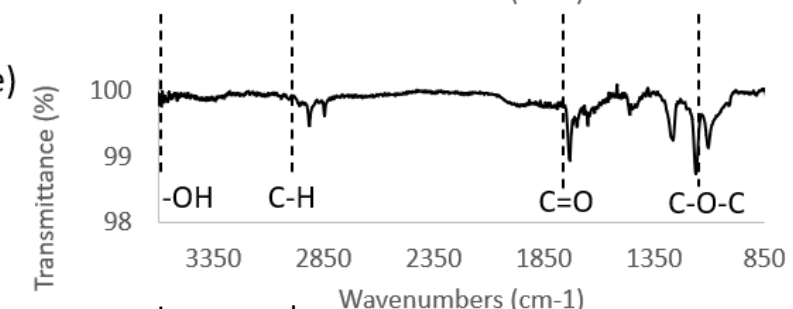

(f)

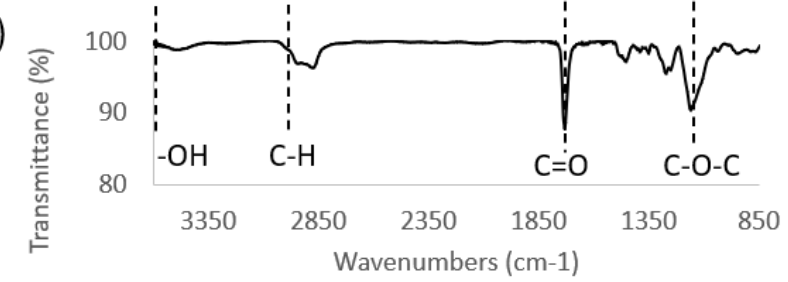

Supplementary Figure 3. Br3d XPS spectra for PPX-HM (yellow), CBP (blue), and DBP (gray)

(a) before, and (b) after reaction with the ATRP initiator. The green and dark blue lines in (b) indicate the corresponding for PPX-HM, while the flat blue and grey lines indicate no Br on CBP or DBP. (c) The XPS spectra after ATRP polymerization. The FTIR spectra of PPX-HM are given (d) before and (e) after the reaction with the ATRP initiator. (f) FTIR spectra of PEGMA polymer brushes on the PPX-HM surfaces after ATRP. 
(a)

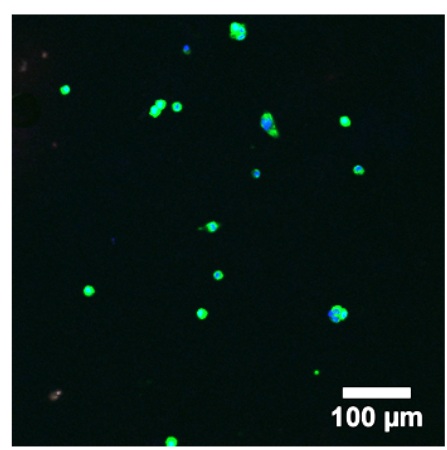

(b)

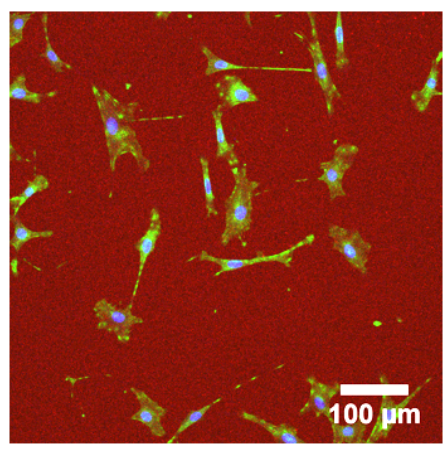

(c)

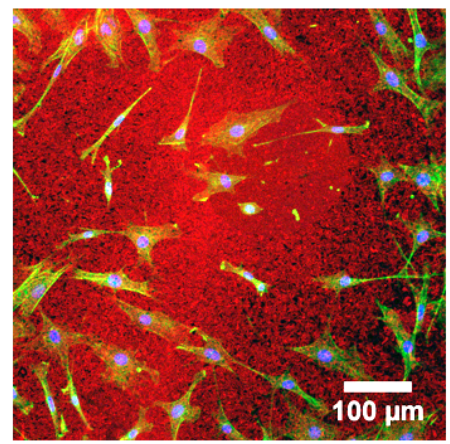

Blue $=$ Cell Nuclei, Red $=$ Fibronectin, Green $=$ F-actin

Supplementary Figure 4. Fibronectin and cells adhered to neat layers of (a) PEGMA polymer brushes, (b) DBP, and (c) CBP. As expected, no fibronectin attaches to the polymer brushes and there is limited cell attachment and spreading. Both DBP and CBP allow for the attachment of fibronectin and the attachment and spreading of cells. Colors are as defined in Supplementary Figure 4. 
(a)

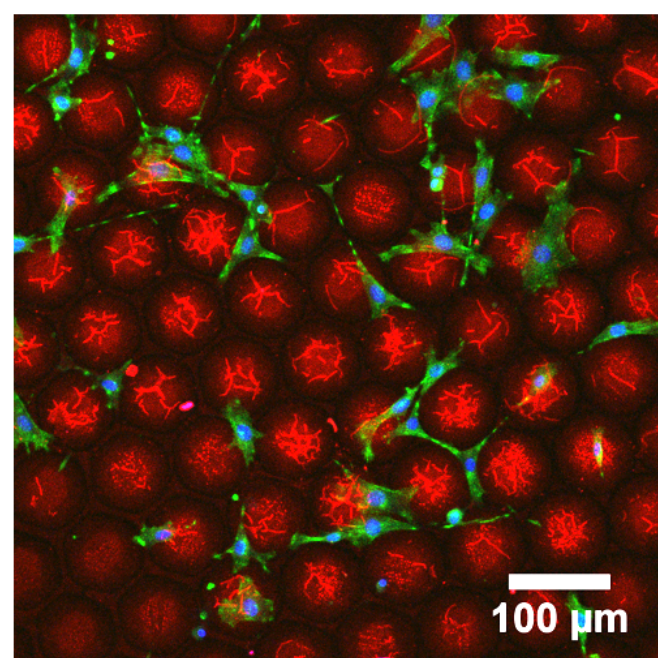

(b)

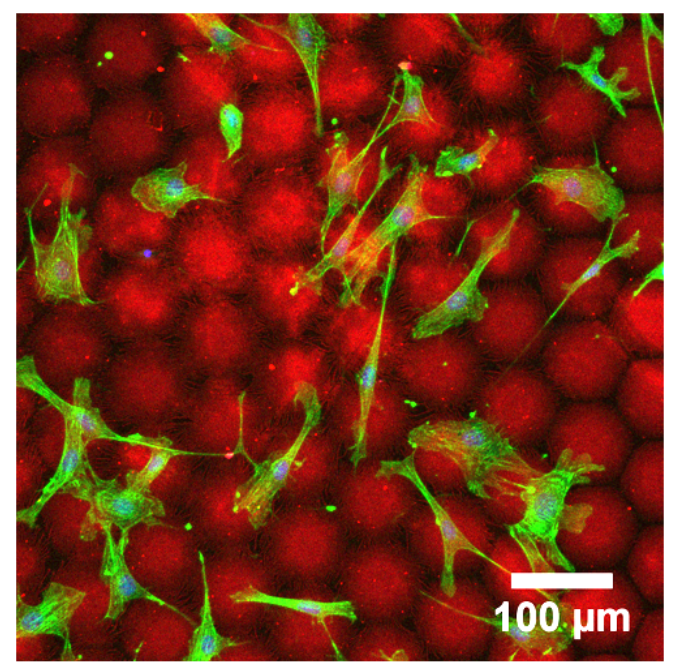

Blue $=$ Cell Nuclei, Red $=$ Fibronectin, Green $=$ F-actin

Supplementary Figure 5. Fibronectin and cells patterned onto substrates without growing PEGMA polymer brushes. (a) DBP-patterned substrates show some selective attachment of fibronectin, but no selective attachment of cells. Fibronectin is bright red, distinguished from the autofluorescence of DBP in the background. (b) CBP-patterned substrates show selective attachment of fibronectin but not cells. Colors are as defined in Supplementary Figure 4. 
(a)

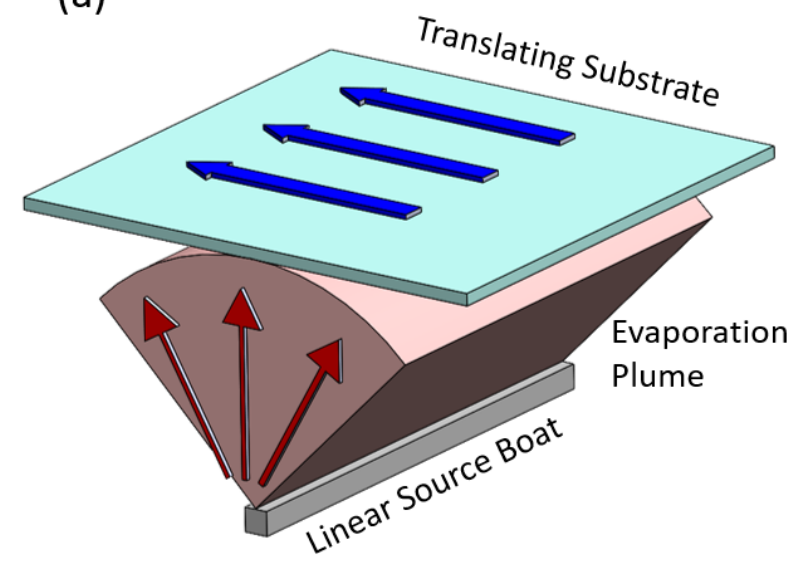

(b)

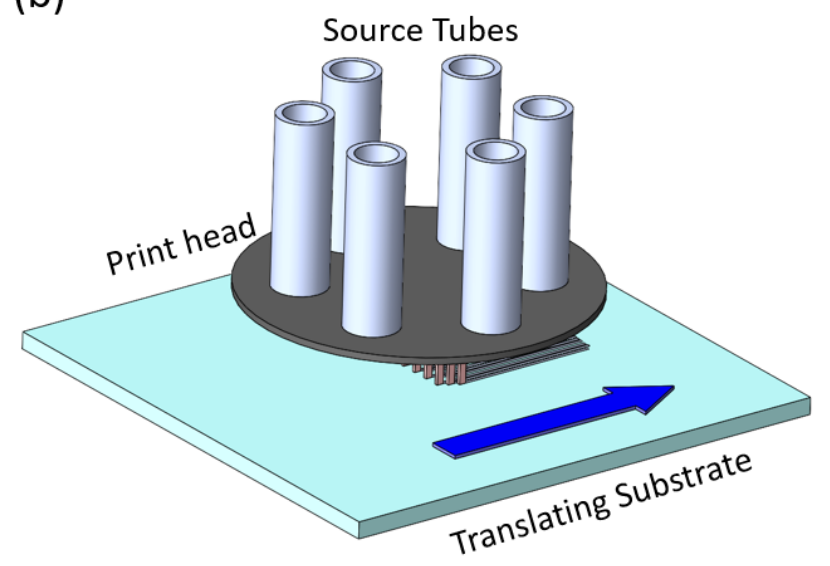

Supplementary Figure 6. (a) Schematic of large-scale deposition by vacuum thermal evaporation. The evaporation plume forms a semi-cylindrical shape, depositing on a substrate as it translates perpendicular to the longer direction of the linear source boat. (b) Schematic of OVJP deposition.

\section{REFERENCES}

(1) S. Liang; J. Wu; H. Tian; L. Zhang; J. Xu. High-Strength Cellulose/Poly(Ethylene Glycol) Gels. ChemSusChem 2008, 558-563. 